\title{
A measurement of the forward-backward charge asymmetry in hadronic decays of the $\mathrm{Z}^{0}$
}

\author{
OPAL Collaboration
}

P D Acton ${ }^{\mathrm{a}}$, G Alexander ${ }^{\mathrm{b}}, \mathrm{J}$ Allıson ${ }^{\mathrm{c}}, \mathrm{P}$ P Allport ${ }^{\mathrm{d}}, \mathrm{K} \mathrm{J}$ Anderson ${ }^{\mathrm{e}}, \mathrm{S}$ Arcellı ${ }^{\mathrm{f}}$, A Astbury ${ }^{\mathrm{g}}, \mathrm{D}$ Axen $^{\mathrm{h}}$, G Azuelos ${ }^{1,1}, \mathrm{G}$ A Bahan ${ }^{\mathrm{c}}, \mathrm{J}$ T M Baines ${ }^{\mathrm{c}}$, A H Ball ${ }^{\mathrm{J}}, \mathrm{J}$ Banks ${ }^{\mathrm{c}}$,

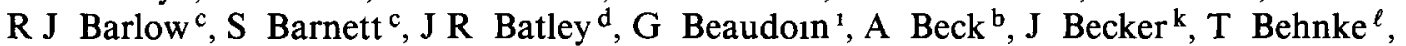
K W Bell ${ }^{\mathrm{m}}, \mathrm{G}$ Bella $^{\mathrm{b}}$, P. Berlich ${ }^{\mathrm{k}}$, S Bethke ${ }^{\mathrm{n}}$, O Biebel ${ }^{\circ}, \mathrm{U}$ Binder $^{\mathrm{k}}$, I J Bloodworth ${ }^{\mathrm{p}}$,

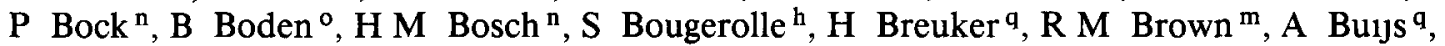
H J Burckhart ${ }^{\mathrm{q}}$, P Capilupp ${ }^{\mathrm{f}}$, R K Carnegie ${ }^{\mathrm{r}}$, A A Carter ${ }^{\mathrm{s}}$, J R Carter ${ }^{\mathrm{d}}$, C Y Chang ${ }^{\mathrm{j}}$, D G Charlton ${ }^{\mathrm{q}}$, P E L Clarke ${ }^{\mathrm{a}}$, I Cohen ${ }^{\mathrm{b}}$, J C Clayton ${ }^{\mathrm{p}}$, W J Collins ${ }^{\mathrm{d}}$, J E Conboy ${ }^{\mathrm{t}}$,

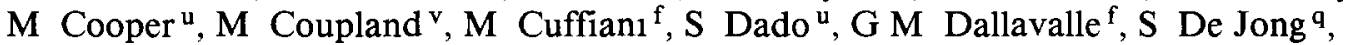
L A del Pozo ${ }^{d}, H$ Deng ${ }^{j}, A$ Dieckmann ${ }^{n}, M$ Dittmar $^{w}$, M S Dixit ${ }^{x}$, E do Couto e Silva ${ }^{y}$, J E Duboscq q, E Duchovni ${ }^{z}, G$ Duckeck ${ }^{n}$, I P Duerdoth ${ }^{c}$, D J P Dumas ${ }^{r}$, P A Elcombe ${ }^{\text {d }}$, P G Estabrooks ${ }^{\mathrm{r}}$, E Etzıon ${ }^{\mathrm{b}}, \mathrm{H} \mathrm{G}$ Evans $^{\mathrm{e}}, \mathrm{F}$ Fabbri ${ }^{\mathrm{f}}$, M Fincke-Keeler ${ }^{\mathrm{g}}, \mathrm{H}$ M Fischer ${ }^{\circ}$,

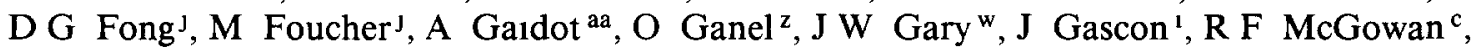
N I Geddes ${ }^{\mathrm{m}}$, C Geich-Gimbel ${ }^{\circ}$, S W Gensler ${ }^{\mathrm{e}}$, F X Gentıt ${ }^{\text {aa }}, \mathrm{G}$ Giacomellı ${ }^{\mathrm{f}}$, V Gibson ${ }^{\mathrm{d}}$, W R Gibson s, J D Gillies ${ }^{\mathrm{m}}$, J Goldberg ", M J Goodrick ${ }^{\mathrm{d}}$, W Gorn w, C Grandif, F C Grant ${ }^{\mathrm{d}}$, J Hagemann ${ }^{\ell}, \mathrm{G} \mathrm{G}$ Hanson ${ }^{\mathrm{y}}, \mathrm{M}$ Hansroul ${ }^{\mathrm{q}}, \mathrm{C} \mathrm{K}$ Hargrove ${ }^{\mathrm{x}}, \mathrm{P}$ F Harrisons,

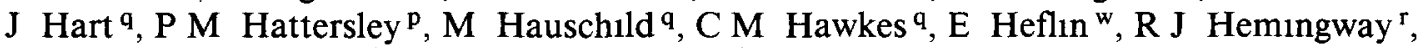

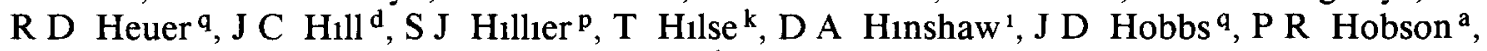
D Hochman ${ }^{2}$, R J Homer ${ }^{p}$, A K Honma ${ }^{g, 1}$, C P Howarth ${ }^{\mathrm{t}}$, R E Hughes-Jones ${ }^{\mathrm{c}}$,

R Humbert ${ }^{k}, P$ Igo-Kemenes ${ }^{n}$, H Ihssen ${ }^{n}$, D C Imrie ${ }^{a}$, A C Janıssen ${ }^{r}$, A Jawahery ${ }^{j}$,

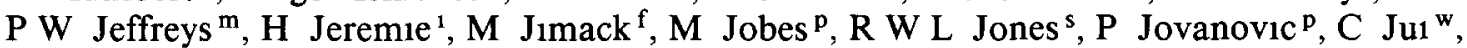
D Karlen ${ }^{\mathrm{r}}$, K Kawagoe ${ }^{\mathrm{ab}}$, T Kawamoto ${ }^{\mathrm{ab}}$, R K Keeler ${ }^{\mathrm{g}}, \mathrm{K}$ G Kellogg ${ }^{\mathrm{j}}$, B W Kennedy ${ }^{\mathrm{t}}$, S Kluth ${ }^{d}$, T Kobayash ${ }^{\text {ab }}$, T P Kokott ${ }^{\circ}$, S Komamıya ${ }^{a b}$, L Kopke $^{\mathrm{q}}$, J F Kral ${ }^{\mathrm{q}}$, R Kowalewskı ${ }^{\mathrm{r}}$, J von Krogh ${ }^{\mathrm{n}}$, J Kroll ${ }^{\mathrm{e}}, \mathrm{M} \mathrm{Kuwano}^{\mathrm{ab}}$, P Kyberd ${ }^{\mathrm{s}}$, G D Lafferty ${ }^{\mathrm{c}}$, F Lamarche $^{1}$, J G Layter $^{w}$, P Le Du ${ }^{\text {aa }}$, P Leblanc ${ }^{1}$, A M Lee ${ }^{\text {, }}$ M H Lehto ${ }^{\text {t }}$, D Lellouch ${ }^{\text {z }}$,

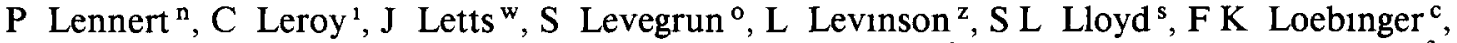

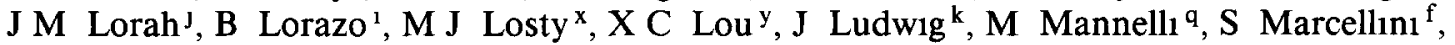
G Marınger ${ }^{\circ}$, C Markus ${ }^{\circ}$, A J Martın ${ }^{\text {s }}$, J P Martın ${ }^{1}$, T Mashımo ${ }^{\text {ab }}$, P Mattıg ${ }^{\circ}$, U Maur ${ }^{\circ}$, J McKenna $^{\mathrm{g}}$, T J McMahon ${ }^{\mathrm{p}}$, J R McNutt ${ }^{\mathrm{a}}$, F Me1jers ${ }^{\mathrm{q}}$, D Menszner ${ }^{\mathrm{n}}$, F S Merritt ${ }^{\mathrm{e}}$, H Mes ${ }^{x}$, A Michelın ${ }^{q}$, R P Middleton ${ }^{m}, G$ Mikenberg $^{z}$, J Mildenberger ${ }^{\mathrm{r}}$, D J Miller ${ }^{\mathrm{t}}$, R Mir ${ }^{\mathrm{y}}$, W Mohr ${ }^{\mathrm{k}}, \mathrm{C}$ Molsan $^{1}$, A Montanar1 ${ }^{\mathrm{f}}$, T Mor1 ${ }^{\mathrm{ab}}, \mathrm{M}$ Mori1 ${ }^{\mathrm{ab}}$, T Mouthuy ${ }^{\mathrm{y}, 2}$, B Nellen ${ }^{\circ}$, H H Nguyen ${ }^{\mathrm{e}}$, M Nozak1 ${ }^{\mathrm{ab}}$, S W O'Neale ${ }^{\mathrm{q}, 3}, \mathrm{~F}$ G Oakham ${ }^{\mathrm{x}}$, F Odorici ${ }^{\mathrm{f}}$, H O Ogren ${ }^{y}$, C J Oram ${ }^{g, 1}$, M J Oreglia ${ }^{\mathrm{e}}, \mathrm{S}$ Orito $^{\mathrm{ab}}$, J P Pansart ${ }^{\mathrm{aa}}$, B Panzer-Steindel ${ }^{\mathrm{q}}$, P Paschievic1 ${ }^{z}$, G N Patrick ${ }^{m}$, N Paz-Jaoshvil1 ${ }^{b}$, P Pfister ${ }^{k}$, J E Pilcher ${ }^{e}$, D Pitman ${ }^{g}$,

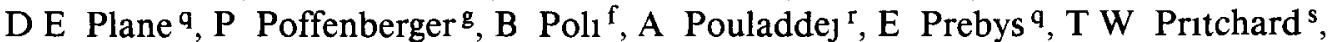

H Przysiezniak ${ }^{1}, G$ Quast $^{\ell}$, M W Redmond ${ }^{\mathrm{e}}, \mathrm{D}$ L Rees ${ }^{\mathrm{p}}, \mathrm{G}$ E Richards ${ }^{\mathrm{c}}$, D Robinson ${ }^{\mathrm{q}}$,

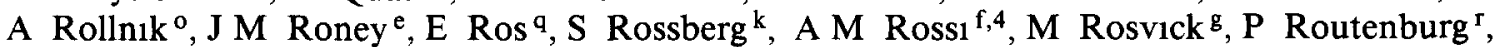
K Runge ${ }^{k}$, O Runolfsson ${ }^{q}$, D R Rust ${ }^{y}, M$ Sasak ${ }^{a b}$, C Sbarra ${ }^{q}$, A D Schalle ${ }^{k}$, O Schalle ${ }^{k}$, W Schappert ${ }^{r}, P$ Scharff-Hansen ${ }^{q}, P$ Schenk ${ }^{g}, H$ von der Schmitt $^{n}, S$ Schreiber $^{\circ}$, 
C Schwick ${ }^{\ell}$, J Schwienıng ${ }^{\circ}$, W G Scott $^{\mathrm{m}}$, M Settles ${ }^{\mathrm{y}}, \mathrm{T}$ G Shears ${ }^{\mathrm{d}}, \mathrm{B}$ C Shen ${ }^{\mathrm{w}}$, C H Shepherd-Themistocleous ${ }^{x}, P$ Sherwood ${ }^{t}$, R Shypit ${ }^{\text {h }}, A$ Simon ${ }^{\circ}, P$ Singh ${ }^{\text {s }}$, G P Sirolı ${ }^{f}$,

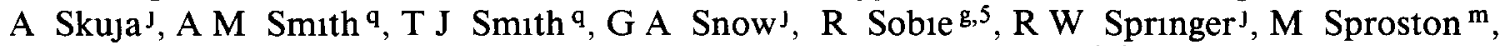

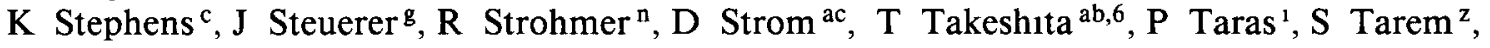

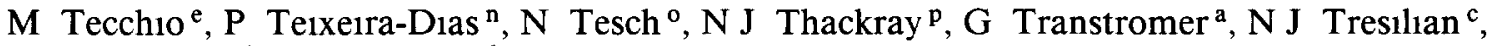
T Tsukamoto $^{\mathrm{ab}}$, M F Turner $^{\mathrm{d}}$, G Tysarczyk-Niemeyer ${ }^{\mathrm{n}}$, D Van den plas ${ }^{1}$, R Van Kooten ${ }^{\mathrm{q}}$, G J VanDalen ${ }^{\text {w }}$, G Vasseur ${ }^{\text {aa }}$, C J Virtue ${ }^{\mathrm{x}}$, A Wagner ${ }^{\ell}$, D L Wagner ${ }^{\mathrm{e}}$, C Wahl ${ }^{\mathrm{k}}$,

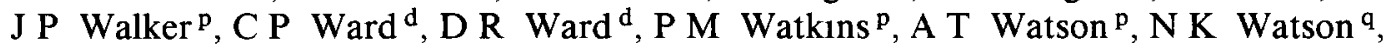
M Weber ${ }^{n}, P$ Weber $^{r}, S$ Weisz ${ }^{q}, P S$ Wells ${ }^{q}, N$ Wermes $^{n}$, M A Whalley ${ }^{p}, G$ W Wilson ${ }^{w}$, J A Wilson ${ }^{\mathrm{p}}, \mathrm{V}-\mathrm{H}$ Winterer ${ }^{\mathrm{k}}, \mathrm{T}$ Wlodek $^{\mathrm{z}}, \mathrm{S}$ Wotton $^{\mathrm{n}}, \mathrm{T} \mathrm{R}$ Wyatt $^{\mathrm{c}}, \mathrm{R}$ Yaar1 $^{\mathrm{z}}, \mathrm{A}$ Yeaman $^{\mathrm{s}}$, G Yekutıelı ${ }^{\mathrm{z}}$, M Yurko ${ }^{1}, \mathrm{~W}$ Zeuner ${ }^{\mathrm{q}}$ and G T Zorn ${ }^{\mathrm{j}}$

a Brunel University, Uxbridge, Middlesex UB8 $3 P H, U K$

b Department of Physics and Astronomy, Tel Aviv University, Tel Aviv 69978, Israel

c Department of Physics, Schuster Laboratory, The University, Manchester M13 9PL, UK

d Cavendish Laboratory, Cambridge CB3 OHE, UK

e Enrico Fermi Institute and Department of Physics, Untversity of Chicago, Chicago, IL 60637, USA

f Dipartimento de Fisica dell' Universita di Bologna and INFN, I-40126 Bologna, Italy

g Department of Physics, University of Victoria, PO Box 3055, Victoria, BC, Canada V8W 3P6

h Department of Physics, University of British Columbia, 6224 Agriculture Road, Vancouver, BC, Canada V6T 1Z1

${ }^{1}$ Laboratotre de Physique Nucleatre, Universite de Montréal, Montreal, Quebec, Canada H3C $3 J 7$

1 Department of Physics and Astronomy, University of Maryland, College Park, MD 20742, USA

k Fakultat fur Physik, Albert Ludwigs Unlversitat, W-7800 Freiburg, FRG

$\ell$ Universitat Hamburg/DESY, II Institut fur Expertmental Physlk, W-2000 Hamburg 52, FRG

m Rutherford Appleton Laboratory, Chilton, Didcot, Oxfordshire OX11 0QX, UK

n Physikalisches Institut, Universitat Heidelberg, W-6900 Heldelberg, FRG

- Physikalisches Institut, Untversitat Bonn, W-5300 Bonn I, FRG

p School of Physics and Space Research, University of Birmingham, Birmingham B15 2TT, UK

q CERN, European Organisation for Particle Physics, CH-1211 Geneva 23, Switzerland

r Department of Physics, Carleton University, Colonel By Drive, Ottawa, Ontarlo, Canada K1S 5B6

sueen Mary and Westfield College, University of London, London E1 4NS, UK

t University College London, London WC1E 6BT, UK

" Department of Physics, Technion-Israel Institute of Technology, Halfa 32000, Israel

$\checkmark$ Birkbeck College, London WCIE $7 H V, U K$

w Department of Physics, University of Callfornia, Riverside, CA 92521, USA

x Centre for Research in Particle Physics, Carleton Unlversity, Ottawa, Ontarlo, Canada K1S $5 B 6$

y Department of Physics, Indiana University, Swain Hall West 117, Bloomington, IN 47405, USA

${ }^{z}$ Nuclear Physics Department, Welzmann Institute of Sclence, Rehovot 76100, Israel

aa DPhPE, CEN-Saclay, F-91191 Gif-sur-Yvette, France

ab International Centre for Elementary Particle Physics and Department of Physics, University of Tokyo, Tokyo 113, Japan and Kobe University, Kobe 657, Japan

ac Untversity of Oregon, Department of Physics, Eugene, OR 97403, USA

Received 21 July 1992

We present a measurement of the forward-backward charge asymmetry in hadronic decays of the $Z^{0}$ using data collected with the OPAL detector at LEP The forward-backward charge asymmetry was measured using a weight function method which gave the number of forward events on a statistıcal basıs In a data sample of 448942 hadronıc $Z^{0}$ decays, we have observed a charge asymmetry of $A_{\mathrm{h}}=0040 \pm 0004$ (stat ) \pm 0006 (syst ) $\pm 0002\left(\mathrm{~B}^{0} \overline{\mathrm{B}}^{0} \mathrm{mix}\right.$ ), takıng into account the effect of $\mathrm{B}^{0} \overline{\mathrm{B}^{0}}$ mixing In the framework of the standard model, this asymmetry corresponds to an effective weak mixIng angle averaged over five quark flavours of $\sin ^{2} \bar{\theta}_{W}=02321 \pm 00017$ (stat ) \pm 00027 (syst) \pm 00009 ( $\mathrm{B}^{0} \overline{\mathrm{B}}^{0} \mathrm{mix}$ ) The result agrees with the value obtained from the $\mathrm{Z}^{0}$ line shape and lepton pair forward-backward asymmetry 


\section{Introduction}

The forward-backward charge asymmetry of fermion pairs from $Z^{0}$ decays is sensitive to the fermion couplings to the $Z^{0}$ The measurement of charged lepton pair asymmetry [1] has been well established and provides a good test of the standard model Here, as a complementary measurement to the lepton sector, the measurement of the quark charge asymmetry, summed over the five quark flavours, is presented

The standard model predicts that the differential cross section for quark pair $(\mathrm{q} \overline{\mathrm{q}})$ production in $\mathrm{e}^{+} \mathrm{e}^{-}$ annihilation at the Born level is

$\frac{\mathrm{d} \sigma_{\mathrm{q}}}{\mathrm{d} \Omega}=\frac{\alpha^{2}}{4 s}\left[C_{0}^{\mathrm{q}}\left(1+\cos ^{2} \theta_{\mathrm{q}}\right)+C_{1}^{\mathrm{q}} \cos \theta_{\mathrm{q}}\right]$,

where

$$
\begin{aligned}
& C_{0}^{\mathrm{q}}=e_{\mathrm{q}}^{2}-8 e_{\mathrm{q}} g_{\mathrm{V}}^{\mathrm{e}} g_{\mathrm{V}}^{\mathrm{q}} \operatorname{Re}(\chi) \\
& \quad+16\left[\left(g_{\mathrm{V}}^{\mathrm{e}}\right)^{2}+\left(g_{\mathrm{A}}^{\mathrm{e}}\right)^{2}\right]\left[\left(g_{\mathrm{V}}^{\mathrm{q}}\right)^{2}+\left(g_{\mathrm{A}}^{\mathrm{q}}\right)^{2}\right]|\chi|^{2}, \\
& C_{1}^{\mathrm{q}}=16\left[-e_{\mathrm{q}} g_{\mathrm{A}}^{\mathrm{e}} g_{\mathrm{A}}^{\mathrm{q}} \operatorname{Re}(\chi)+8 g_{\mathrm{V}}^{\mathrm{e}} g_{\mathrm{V}}^{\mathrm{q}} g_{\mathrm{A}}^{\mathrm{e}} g_{\mathrm{A}}^{\mathrm{q}}|\chi|^{2}\right]
\end{aligned}
$$

and

$\chi=\frac{G_{\mathrm{F}} M_{\mathrm{Z}}^{2}}{8 \pi \alpha \sqrt{2}} \frac{s}{s-M_{\mathrm{Z}}^{2}+1 M_{\mathrm{Z}} \Gamma_{\mathrm{Z}}}$

In the above equations, $\theta_{\mathrm{q}}$ is the angle between the directions of the incoming electron and the outgoIng quark ${ }^{\# 1}, e_{\mathrm{q}}$ is the quark charge, $g_{\mathrm{V}}^{\mathrm{e}}$ and $g_{\mathrm{V}}^{\mathrm{q}}$ are the vector, and $g_{\mathrm{A}}^{\mathrm{e}}$ and $g_{\mathrm{A}}^{\mathrm{q}}$ are the axial vector coupling constants of the electron and quark to the $Z^{0}$, respectively The quark couplings for each flavour $q$ are given by

1 Also at TRIUMF, Vancouver, Canada V6T 2A3

2 Present address Centre de Physique des Particules de Marseille, Faculte des Sciences de Luminy, Marseille, France

3 On leave from Bırmıngham Unıversity, Bırmıngham B15 2TT, UK

4 Present address Dipartımento dı Fisıca, Unıversita della Calabria and INFN, I-87036 Rende, Italy

5 And IPP, McGill University, High Energy Physics Department, 3600 University Str, Montreal, Quebec, Canada H3A 2T8

6 Also at Shınshu Unıversity, Matsumoto 390, Japan

\#1 Throughout this analysis, all polar angles are measured with respect to the incoming electron direction
$g_{\mathrm{v}}^{\mathrm{q}}=\frac{I_{3}^{\mathrm{q}}}{2}-2\left|e_{\mathrm{q}}\right| \sin ^{2} \bar{\theta}_{\mathrm{W}}(\mathrm{q})$,

$g_{\mathrm{A}}^{\mathrm{q}}=\frac{l_{3}^{\mathrm{q}}}{2}$

where $I_{3}^{\mathrm{q}}$ is the third component of the weak isospin of the quark and $\sin ^{2} \bar{\theta}_{\mathrm{W}}$ is its effective weak mixing angle, which can be expressed as

$\sin ^{2} \bar{\theta}_{\mathrm{W}}(\mathrm{q}) \equiv\left(1-\frac{M_{\mathrm{W}}^{2}}{M_{\mathrm{Z}}^{2}}\right) \operatorname{Re}\left[\kappa_{\mathrm{q}}\left(M_{\mathrm{Z}}^{2}\right)\right]$,

with the form factor $\kappa_{\mathrm{q}}\left(M_{\mathrm{Z}}^{2}\right)$ described in ref [2] This form factor is also dependent on the top quark mass, the Higgs mass and the mass of the given quark, and is slightly different for each quark flavour

The forward-backward charge asymmetry for each quark flavour is defined as

$$
\begin{aligned}
A_{\mathrm{q}} & \equiv \frac{\int_{\cos \theta_{\mathrm{q}}>0}\left(\mathrm{~d} \sigma_{\mathrm{q}} / \mathrm{d} \Omega\right) \mathrm{d} \Omega-\int_{\cos \theta_{\mathrm{q}}<0}\left(\mathrm{~d} \sigma_{\mathrm{q}} / \mathrm{d} \Omega\right) \mathrm{d} \Omega}{\int\left(\mathrm{d} \sigma_{\mathrm{q}} / \mathrm{d} \Omega\right) \mathrm{d} \Omega} \\
& =3 C_{1}^{\mathrm{q}} / 8 C_{0}^{\mathrm{q}},
\end{aligned}
$$

and the fraction of hadronic decays into that flavour is $f_{\mathrm{q}}=C_{0}^{\mathrm{q}} / \sum_{t} C_{0}^{t}$

For the total hadronic charge asymmetry, we define the production polar angle $\theta_{\mathrm{h}}$ to be between the direction of the incoming electron and that of the outgoing parton with negative charge The hadronic asymmetry is then

$A_{\mathrm{h}}=f_{\mathrm{d}} A_{\mathrm{d}}-f_{\mathrm{u}} A_{\mathrm{u}}+f_{\mathrm{s}} A_{\mathrm{s}}-f_{\mathrm{c}} A_{\mathrm{c}}+f_{\mathrm{b}} A_{\mathrm{b}}$

Note that the negative signs arise for the positively charged quarks because of our definition of the asymmetry

As mentioned, the effective weak mixing angle is slightly different for each quark flavour We will interpret our results in terms of the average effectıve weak mixing angle over the five avallable quark flavours for a value of the top quark mass which gives a set of $\sin ^{2} \bar{\theta}_{\mathrm{W}}$ values that reproduces our measured hadronic asymmetry

The determination of the direction and charge of the underlying partons from the final state particles is the largest experimental challenge of this analysis It will be shown that its dependence on the Monte Carlo modelling is controllable 
Several experiments at PEP, PETRA and TRISTAN have reported their measurements of the inclusive hadronic asymmetry [3] While these lower energy experiments essentially measure the axial couplings of the quarks to the $Z^{0}$, the asymmetry at the $\mathrm{Z}^{0}$ resonance is sensitive to the vector coupling, and therefore to the effective weak mixing angles

The ALEPH and DELPHI collaborations have recently published results on the measurement of the charge asymmetry in hadronic $Z^{0}$ decays [4]

\section{The OPAL detector and event selection}

The OPAL detector has been described in detail elsewhere [5] It is a multipurpose detector covering almost the entire solid angle around the interaction point Its main components are a system of central trackıng chambers inside a magnetic field of $0435 \mathrm{~T}$, an electromagnetic calorımeter, a hadron calorımeter and an outer layer of muon chambers

For this analysıs the central trackıng chambers and the electromagnetic calorimeter were used The central tracking system provides up to 183 space points per track and close to $100 \%$ trackıng efficiency for charged tracks in the polar angle range defined by $|\cos \theta|<092$ High energy tracks are measured with a momentum resolution $\sigma(p) / p^{2} \approx 15 \times 10^{-3} \mathrm{GeV}^{-1}$ The electromagnetic calorimeter consists of a cylındrical array of 9440 lead glass blocks in the barrel region $(|\cos \theta|<082)$ and 2264 lead glass blocks in the two end cap regions $(081<|\cos \theta|<098)$ It has a typical energy resolution of $\Delta E / E \approx 3 \%$ for $E \approx$ $45 \mathrm{GeV}$

Monte Carlo studies were performed using a detailed simulation of the OPAL detector [6], which modelled detector geometry and material as well as resolution and efficiency The events were generated using the Lund parton shower model, as implemented in the JETSET [7] Monte Carlo package

The data used in this analysis correspond to an integrated luminosity of $21 \mathrm{1} \mathrm{pb}^{-1}$, collected in 1990 and 1991 The selection of hadronic $Z^{0}$ decays was based on energy clusters in the electromagnetic calorimeter and charged track multiplicity as described in ref [1] The sample consists of 448942 hadronic $Z^{0}$ decays

For this analysis, the track definition was tightened by demanding at least 40 measured space points, and the following additional criteria were required to be satisfied

(1) The sphericity, $S$, was calculated using charged tracks only Only events with $S<012$ were selected This cut suppressed events with hard gluon radiation and selected events where the sphericity axis closely approximated the original quark direction, however, it was loose enough that the fraction of $b \bar{b}$ events was not significantly reduced, compared to lighter quarks (11) The polar angle of the sphericity axis, $\theta_{\text {sph }}$, was required to be in the range $\left|\cos \theta_{\mathrm{sph}}\right|<08$, so that the event was contained well in the detector acceptance

(111) The event was divided by the plane perpendicular to the sphericity axis At least three tracks were required in each hemisphere

(iv) Any event with at least one track with momentum greater than the beam energy was removed Such tracks were caused by reconstruction problems and could bias the jet charge determination

After applying the additional selection criteria described above, the resulting data sample contained 258869 events

\section{Method of charge assignment}

The charge of the quark is reflected to some extent by the charges of the leading particles in the resulting jet Rather than determınıng the jet charge on an event by event basis, we have chosen a statistical method to determine the asymmetry We have adopted the weight function method described in ref [8], which was previously used in the analysis of the JADE Collaboration [3] This method allows an efficient use of the data and results in a high statistical sensitivity

The group of tracks contained in each sphericity hemisphere was regarded as a "jet", and the sphericity axis was taken as the jet direction To determine the jet charge, we chose the following variable to describe each track

$z=q\left(\frac{\left|p_{\|}\right|}{E_{\text {beam }}}\right)^{\kappa}$,

where $q$ and $p_{\|}$are the track charge and momentum component parallel to the sphericity axis, respectively, and $E_{\text {beam }}$ is the beam energy We chose the value $\kappa=$ 1 for this analysis As a cross-check, the value $\kappa=0$ was used, this will be discussed below 

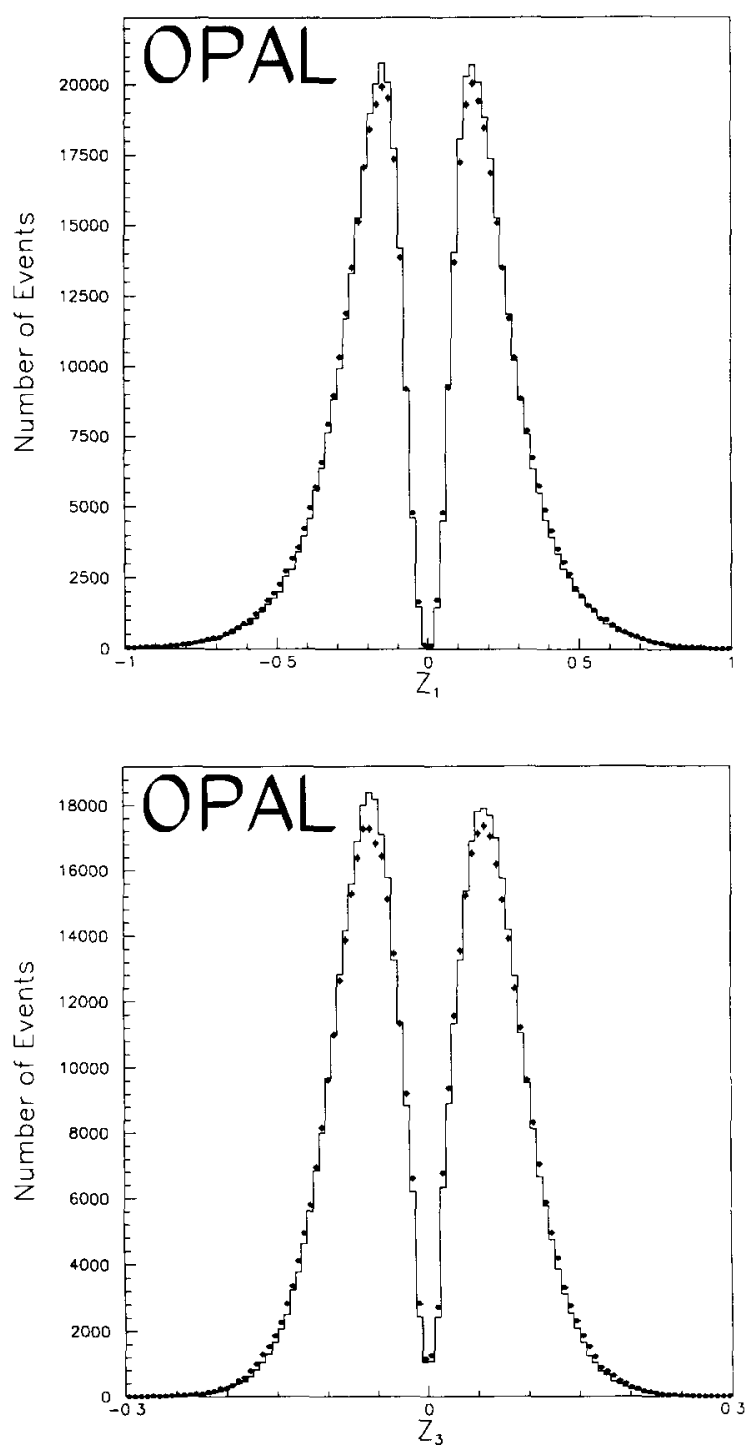

In each jet of the event, we selected the three charged tracks ${ }^{\# 2}$ which had the largest $\left|p_{\|}\right|$in that jet They were ordered such that $\left|p_{\|}^{(1)}\right| \geqslant\left|p_{\|}^{(2)}\right| \geqslant\left|p_{\|}^{(3)}\right|$ and $z_{1}$ was calculated for each $p_{\|}^{(l)}$ In fig 1 , the distributions of $z_{1}, z_{2}$ and $z_{3}$ in the data are compared with the Monte Carlo data with full detector simulation Fig 2 shows the distributions of $z_{1}, z_{2}$

\#2 The limitation to the leading three partıcles per jet is a compromise between the charge information used in each event and the Monte Carlo statistics needed to construct the weight function, as described below

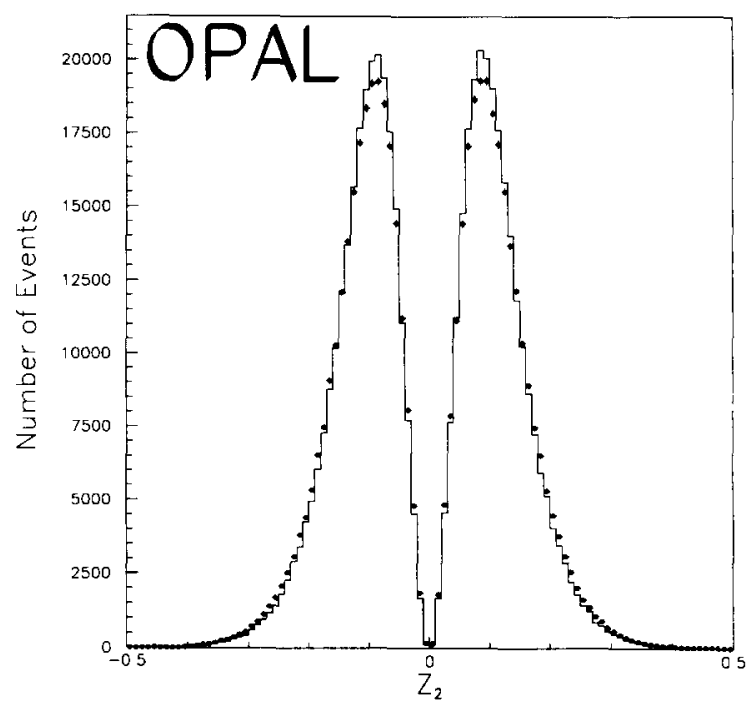

Fig 1 Distribution of $z_{1}, z_{2}$, and $z_{3}$ The cross points represent the data and the line histogram the Monte Carlo prediction with full detector simulation Note that the horizontal scale in each figure is different

and $z_{3}$ for jets which originated from the positive quarks in the Monte Carlo events There is a clear asymmetry in each distribution In addition, there exist correlations among these $z_{i}$ variables

We created the weight function by using $z_{1}, z_{2}$ and $z_{3}$ distributions for the Monte Carlo events in the following way We designated the jet in the forward hemisphere as the forward jet and that in the opposite hemisphere as the backward jet The distributions of the $z_{l}$ variables for the forward jet $\left(z_{l}^{\mathrm{F}}, l=\right.$ $1,2,3)$ and the backward jet $\left(z_{1}^{\mathrm{B}}, l=1,2,3\right)$ were 

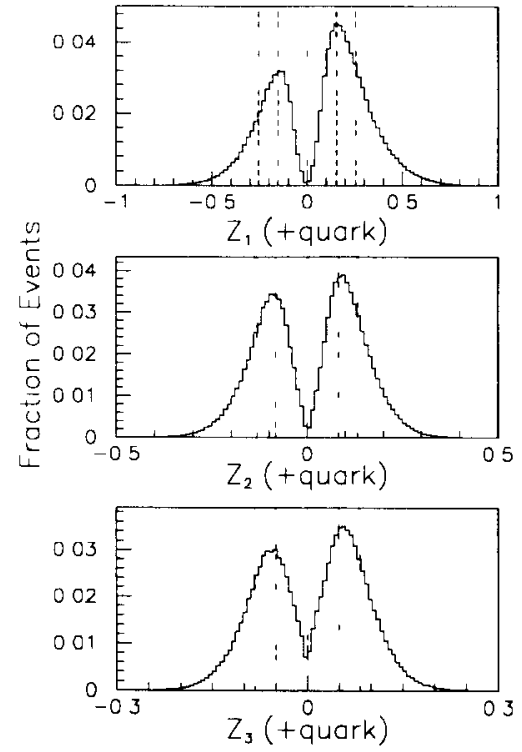

Fig 2 Distribution of $z_{1}, z_{2}$, and $z_{3}$ for the positive partons in Monte Carlo events The binning used to form the weight function is indicated by dashed lines

obtained for Monte Carlo events in which the negative parton travelled in the forward direction (forward events) We termed this six-dimensional distribution $f_{\mathrm{F}}\left([z]^{\mathrm{F}},[z]^{\mathrm{B}}\right)$, where $[z]$ denotes $\left(z_{1}, z_{2}, z_{3}\right)$ The same distribution for events in which the negative parton travelled in the backward direction (backward events $)$ was also obtained and termed $f_{\mathrm{B}}\left([z]^{\mathrm{F}},[z]^{\mathrm{B}}\right)$

Obtaining an accurate six-dimensional distribution requires an extremely large number of Monte Carlo events The task is simplified if we assume that fragmentation of one jet is independent from that of the opposite jet We can then decompose the sixdimensional distribution into two three-dimensional distributions as

$$
\begin{aligned}
& f_{\mathrm{F}}\left([z]^{\mathrm{F}},[z]^{\mathrm{B}}\right)=f_{-}\left([z]^{\mathrm{F}}\right) f_{+}\left([z]^{\mathrm{B}}\right), \\
& f_{\mathrm{B}}\left([z]^{\mathrm{F}},[z]^{\mathrm{B}}\right)=f_{+}\left([z]^{\mathrm{F}}\right) \quad f_{-}\left([z]^{\mathrm{B}}\right),
\end{aligned}
$$

where $f_{-}\left(f_{+}\right)$is the probability that the combination $[z]$, describing the three leading particles in a particular hemisphere, was produced by a negatively (positively) charged parton This decomposition greatly reduces the number of Monte Carlo events required
The resulting distributions are then used to form the weight function

$$
\begin{aligned}
& W\left([z]^{\mathrm{F}},[z]^{\mathrm{B}}\right) \\
& =\frac{f_{\mathrm{F}}\left([z]^{\mathrm{F}},[z]^{\mathrm{B}}\right)}{f_{\mathrm{F}}\left([z]^{\mathrm{F}},[z]^{\mathrm{B}}\right)+f_{\mathrm{B}}\left([z]^{\mathrm{F}},[z]^{\mathrm{B}}\right)} \\
& =\frac{f_{-}\left([z]^{\mathrm{F}}\right) f_{+}\left([z]^{\mathrm{B}}\right)}{f_{-}\left([z]^{\mathrm{F}}\right) f_{+}\left([z]^{\mathrm{B}}\right)+f_{+}\left([z]^{\mathrm{F}}\right) f_{-}\left([z]^{\mathrm{B}}\right)}
\end{aligned}
$$

The weight function defined above represents the probability that the negative parton travelled in the forward direction

It is important to note that the choice of different weight functions can lead to different sensitivities to the measured quantity, but does not bias the result

A simple simulation program, which smeared the particle momenta given by the event generator with the measured detector resolutions, was used to produce a sample of $10^{6}$ Monte Carlo events and to calculate the weight function With each $z_{l}$ distribution divided into six bins, the functions, $f_{+}$and $f_{-}$, were represented as three-dimensional histograms The bin sizes were chosen so that the number of entries in each bin was approximately equal The binning is indicated in fig 2 The weight function was then formed according to eq (1) and smoothing was applied to reduce the statistical fluctuations

A sample of $N$ real events consists of $N_{\mathrm{F}}$ forward events and $N_{\mathrm{B}}$ backward events The weighted number of events is given by

$N \bar{W}=\sum_{l}^{N} W_{l}=N_{\mathrm{F}} \bar{W}_{\mathrm{F}}+N_{\mathrm{B}} \quad \bar{W}_{\mathrm{B}}$,

where $\bar{W}$ is the average of the weight for all the events and $\bar{W}_{\mathrm{F}}$ and $\bar{W}_{\mathrm{B}}$ are the average weights for the forward and backward events, respectively Since $N=$ $N_{\mathrm{F}}+N_{\mathrm{B}}$ holds, we can get the number of forward events as

$N_{\mathrm{F}}=N \frac{\bar{W}-\bar{W}_{\mathrm{B}}}{\bar{W}_{\mathrm{F}}-\bar{W}_{\mathrm{B}}}$

The remainıng $N_{\mathrm{B}}=N-N_{\mathrm{F}}$ events were the backward events

We have divided the real and Monte Carlo event samples into $\left|\cos \theta_{\text {sph }}\right|$ bins of width 02 For each 
Table 1

Average weights of each quark for forward and backward events in each $\left|\cos \theta_{\text {sph }}\right|$ bin

\begin{tabular}{llll}
\hline & $\left|\cos \theta_{\mathrm{sph}}\right|$ & $\bar{W}_{\mathrm{F}}$ & $\bar{W}_{\mathrm{B}}$ \\
\hline d-quark & $00-02$ & $05627 \pm 00029$ & $04368 \pm 00028$ \\
& $02-04$ & $05806 \pm 00027$ & $04191 \pm 00026$ \\
& $04-06$ & $05791 \pm 00026$ & $04215 \pm 00023$ \\
& $06-08$ & $05762 \pm 00024$ & $04226 \pm 00021$ \\
u-quark & $00-02$ & $06093 \pm 00032$ & $03978 \pm 00033$ \\
& $02-04$ & $06213 \pm 00029$ & $03744 \pm 00031$ \\
& $04-06$ & $06294 \pm 00026$ & $03732 \pm 00028$ \\
& $06-08$ & $06281 \pm 00024$ & $03756 \pm 00026$ \\
s-quark & $00-02$ & $05830 \pm 00030$ & $04148 \pm 00033$ \\
& $02-04$ & $06010 \pm 00028$ & $03982 \pm 00026$ \\
& $04-06$ & $06036 \pm 00026$ & $03996 \pm 00023$ \\
& $06-08$ & $06020 \pm 00024$ & $03987 \pm 00021$ \\
c-quark & $00-02$ & $05414 \pm 00032$ & $04661 \pm 00031$ \\
& $02-04$ & $05503 \pm 00030$ & $04568 \pm 00031$ \\
& $04-06$ & $05461 \pm 00027$ & $04551 \pm 00029$ \\
& $06-08$ & $05451 \pm 00024$ & $04543 \pm 00027$ \\
b-quark & $00-02$ & $05932 \pm 00030$ & $04084 \pm 00029$ \\
& $02-04$ & $06194 \pm 00027$ & $03743 \pm 00025$ \\
& $04-06$ & $06196 \pm 00025$ & $03798 \pm 00023$ \\
& $06-08$ & $06227 \pm 00024$ & $03791 \pm 00021$ \\
\hline
\end{tabular}

$\left|\cos \theta_{\mathrm{sph}}\right|$ bin, the average weights, $\bar{W}_{\mathrm{F}}$ and $\bar{W}_{\mathrm{B}}$, for the forward and backward events were calculated for the Monte Carlo events with full detector simulation

$\bar{W}_{\mathrm{F}}=\frac{1}{N_{\mathrm{F}}^{\mathrm{MC}}} \sum_{i=1}^{N_{\mathrm{F}}^{\mathrm{MC}}} W_{l}, \quad \bar{W}_{\mathrm{B}}=\frac{1}{N_{\mathrm{B}}^{\mathrm{MC}}} \sum_{i=1}^{N_{\mathrm{B}}^{\mathrm{MC}}} W_{l}$

Here the summation is over all $N_{\mathrm{F}}^{\mathrm{MC}}$ forward events ( $N_{\mathrm{B}}^{\mathrm{MC}}$ backward events) which enter this $\left|\cos \theta_{\text {sph }}\right|$ bin, and $W_{l}=W\left([z]_{l}^{\mathrm{F}},[z]_{l}^{\mathrm{B}}\right)$ is the weight of the $l$ th event The resulting average weights for each quark for each $\left|\cos \theta_{\text {sph }}\right|$ bin are listed in table 1 The number of forward events is determined for each $\left|\cos \theta_{\mathrm{sph}}\right|$ bin, using $\bar{W}_{\mathbf{F}}$ and $\bar{W}_{\mathbf{B}}$ from the Monte Carlo calculation and $N$ and $\bar{W}$ are measured from the data

In the case of the forward-backward charge asymmetry in hadronic events, the number of forward events and backward events occur randomly with a binomial distribution In this case, the statistical error on $N_{\mathrm{F}}$ contains an additional term due to nonperfect separation of the event classes by the weight function and is given by

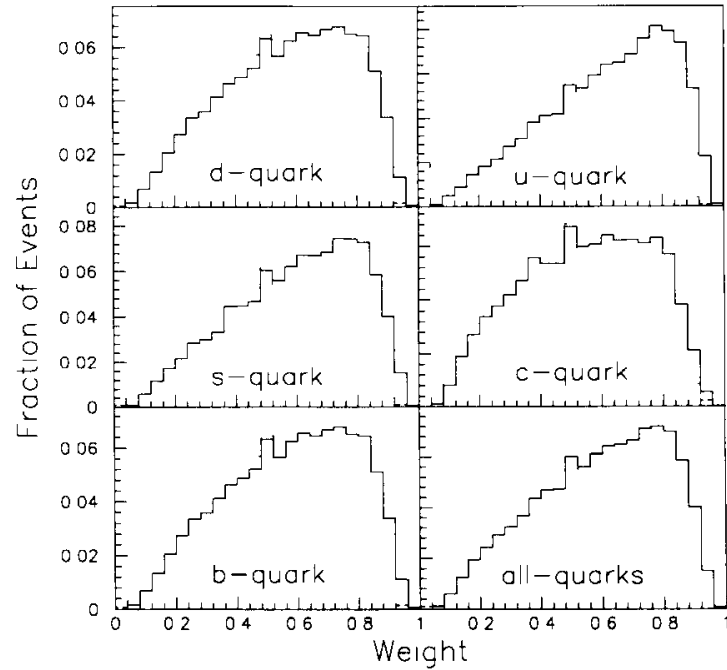

Fig 3 The Monte Carlo distributions of the weights for each quark The solıd lines show the forward events and the dashed lines, the backward events The distribution for all quarks is obtained by adding the weights of the five quark flavours to produce an average charge asymmetry on the parton level of 0040

$\sigma_{\text {stat }}=\sqrt{\frac{N_{\mathrm{F}} N_{\mathrm{B}}}{N}+\frac{N_{\mathrm{F}} \sigma_{\mathrm{F}}^{2}+N_{\mathrm{B}} \sigma_{\mathrm{B}}^{2}}{\left(\bar{W}_{\mathrm{F}}-\bar{W}_{\mathrm{B}}\right)^{2}}}$

Here $\sigma_{\mathrm{F}}$ and $\sigma_{\mathrm{B}}$ are the standard deviations of the weight distributions for the forward and backward Monte Carlo events, respectively (fig 3 ) In addition, there is an error due to the statistical precision of $\bar{W}_{\mathrm{F}}$ and $\bar{W}_{\mathrm{B}}$, which is limited by the number of Monte Carlo events This results in an error on $N_{\mathrm{F}}$ of

$$
\begin{aligned}
\sigma_{\text {stat }}^{\mathrm{MC}} & =\frac{N}{\left(\bar{W}_{\mathrm{F}}-\bar{W}_{\mathrm{B}}\right)^{2}} \\
& \times \sqrt{\left(\bar{W}-\bar{W}_{\mathrm{F}}\right)^{2} \frac{\sigma_{\mathrm{B}}^{2}}{N_{\mathrm{B}}^{\mathrm{MC}}}+\left(\bar{W}-\bar{W}_{\mathrm{B}}\right)^{2} \frac{\sigma_{\mathrm{F}}^{2}}{N_{\mathrm{F}}^{\mathrm{MC}}}}
\end{aligned}
$$

In fig $4 \mathrm{a}$, the distribution of the weights for data is compared with that for Monte Carlo events with a charge asymmetry at the parton level of 004 The enhancement in the weight at 05 is due to events in which both jets have the same charge combination To further check the matching between the data and Monte Carlo events, we define 

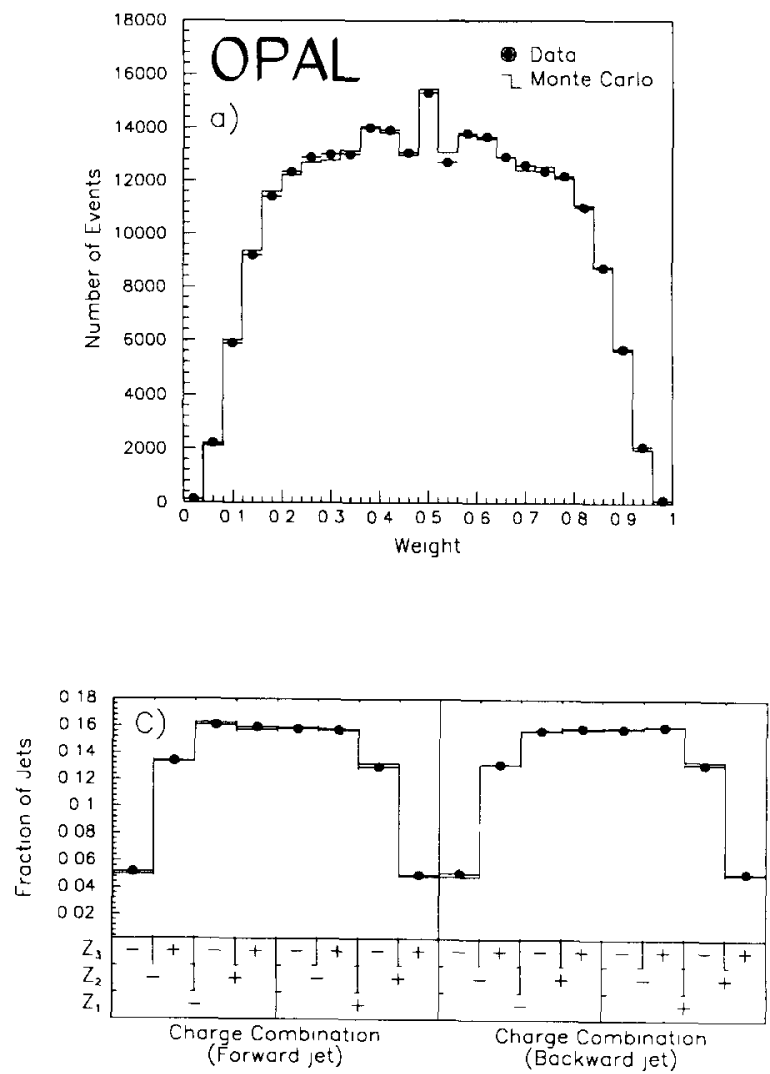

$Q_{\mathrm{F}}=\frac{f_{-}\left([z]^{\mathrm{F}}\right)-f_{+}\left([z]^{\mathrm{F}}\right)}{f_{-}\left([z]^{\mathrm{F}}\right)+f_{+}\left([z]^{\mathrm{F}}\right)}$,

$Q_{\mathrm{B}}=\frac{f_{-}\left([z]^{\mathrm{B}}\right)-f_{+}\left([z]^{\mathrm{B}}\right)}{f_{-}\left([z]^{\mathrm{B}}\right)+f_{+}\left([z]^{\mathrm{B}}\right)}$

Fig $4 \mathrm{~b}$ shows $Q_{\mathrm{F}} Q_{\mathrm{B}}$ for data and Monte Carlo events The charge combination of selected tracks in each jet is shown in fig 4c All of these distributions show the sensitivity of the weights to the quark charge The $1 \mathrm{~m}$ pact of the differences between data and Monte Carlo on the result of the measurement will be discussed later

In the standard model, different quark flavours are expected to have different asymmetries, all of which depend on $\sin ^{2} \bar{\theta}_{\mathrm{w}}$ In particular, for our definition of the asymmetry, the u-type ( $u$ and $c$ ) and the $d-$ type ( $\mathrm{d}, \mathrm{s}$ and $\mathrm{b}$ ) quarks contribute to the total asymmetry with opposite sign This can potentially introduce a bias because different quark flavours fragment

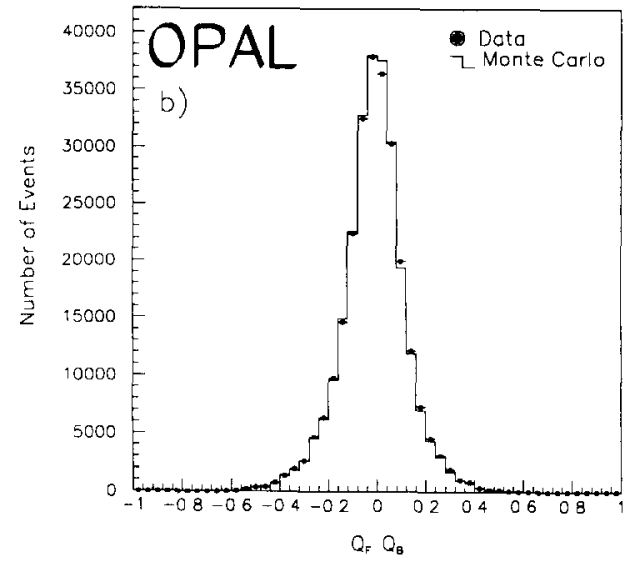

Fig 4 Comparison of data (closed circle) and Monte Carlo (histogram) events (a) shows the weights The enhancement at 05 is due to events in which both jets have the same charge combination (b) shows $Q_{\mathrm{F}} Q_{\mathrm{B}}$ and (c) shows the charge combinations of the selected tracks in each jet

differently, producing different weight function distributions and hence different values for $\bar{W}_{\mathrm{F}}$ and $\bar{W}_{\mathrm{B}}$, as shown in table 1 Obtaining the correct average weight therefore depends on having the correct quark composition for the events with the true asymmetry for each quark Since both the quark composition and the asymmetry depend on $\sin ^{2} \bar{\theta}_{\mathrm{W}}$, the determination of the asymmetry is an iterative procedure This will be discussed in more detail shortly

The events were generated for only one value of $\sin ^{2} \bar{\theta}_{\mathrm{w}}, 1 \mathrm{e}$ one value of the total quark-antiquark charge asymmetry Different asymmetries were then simulated by adjusting the relative numbers of forward and backward events for each of the five quark flavours and by changing the corresponding flavour mixtures as predicted by the standard model, for var1ous values of $\sin ^{2} \bar{\theta}_{\mathrm{W}}$

In order to obtain the charge asymmetry at the quark level, one must correct for the effect of $\mathrm{B}^{0} \overline{\mathrm{B}^{0}}$ 
mixing, which has the effect of reducing the observed asymmetry This effect was estımated by implementing $\mathrm{B}^{0} \overline{\mathrm{B}^{0}}$ mixing in the Monte Carlo event generator Note that the size of the correction is approximately proportional to the size of the $\mathrm{B}^{0} \overline{\mathrm{B}^{0}}$ asymmetry Assuming standard model behaviour, the $\mathrm{B}^{0} \overline{\mathrm{B}^{0}}$ asymmetry is related to the effective weak mixing angle, which also gives the total charge asymmetry Therefore, the effect of $\mathrm{B}^{0} \overline{\mathrm{B}^{0}}$ mixing has been evaluated using a Monte Carlo in which the asymmetry has been tuned to match the observed data, $1 \mathrm{e}$

$A_{\mathrm{h}}=A_{\mathrm{h}}^{0}+\Delta A_{\mathrm{B}^{0} \overline{\mathrm{B}^{0}} \mathrm{~m} \mathrm{xx}}$,

where $A_{\mathrm{h}}^{0} 1 \mathrm{~s}$ the observed asymmetry in the data without correction for $\mathrm{B}^{0} \overline{\mathrm{B}^{0}}$ mixing $A_{\mathrm{h}}$ is the measured charge asymmetry with a correction for $\mathrm{B}^{0} \overline{\mathrm{B}^{0}}$ mixing, expressed as $\Delta A_{\mathrm{B}^{0} \overline{\mathrm{B}}^{0} \mathrm{mix}}$ The $\mathrm{B}^{0} \overline{\mathrm{B}^{0}}$ mixing correction term is determined from the Monte Carlo by comparıng the asymmetry corrected and uncorrected for this effect, while adjusting the corrected Monte Carlo asymmetry to the measured asymmetry The following experımentally measured mixing ratıo was used [9]

$\chi=0145 \pm 0045$

Here, $\chi$ can be broken down into contributions from $B_{d}^{0}$ and $B_{s}^{0}$ mesons as

$\chi=f_{\mathrm{d}} \chi_{\mathrm{d}}+f_{\mathrm{s}} \chi_{\mathrm{st}}$

where $\chi_{l}$ is the mixing ratio for $\mathrm{B}_{l}^{0}$ mesons and $f_{l}$ is the fraction of the primary $b$ quark fragmenting into that meson To estimate the effect of the $\mathrm{B}^{0} \overline{\mathrm{B}^{0}}$ mixing, we used the above value for the total mixing and a value for $\chi_{\mathrm{d}}$ of $017 \pm 004$, as measured by the ARGUS and CLEO experıments [10] Assuming $f_{\mathrm{d}}=0375 \pm$ 0050 and $f_{\mathrm{s}}=0150 \pm 0050$, we observed a shift in the measured asymmetry of Monte Carlo events of $\Delta A_{\mathrm{B}^{0} \overline{\mathrm{B}}_{\text {mix }}}=00044 \pm 00015$ for a charge asymmetry of 004 at parton level Here, the error was obtained by allowing the products $\left(f_{\mathrm{d}} \chi_{\mathrm{d}}\right)$ and $\left(f_{\mathrm{s}} \chi_{\mathrm{s}}\right)$ to vary to all combinations of extreme values allowed by the quoted numbers and accounting for uncertainties in the decay mode

\section{Measurement of the charge asymmetry}

Since the efficiency of event selection is dependent on $\left|\cos \theta_{\text {sph }}\right|$, and in order to relate the sphericity axis to the parton direction, an acceptance correction was made for each $\cos \theta_{\mathrm{sph}}$ bin After applying the acceptance correction, the angular distribution of the negatively charged quarks, $\cos \theta_{\mathrm{h}}$, was obtained The corrected number of events for the $\imath$ th bin, $N_{l}^{\text {corr }}$, was calculated by

$N_{l}^{\text {corr }}=\frac{N_{l, \mathrm{MC}}^{\mathrm{gen}}}{N_{l, \mathrm{MC}}^{\mathrm{accpt}}} N_{l}^{\mathrm{accp},}$

where $N_{l}^{\mathrm{accpt}}$ and $N_{i, \mathrm{MC}}^{\mathrm{accpt}}$ are the numbers of data and Monte Carlo events, respectively, which pass the selection, and $N_{l \mathrm{MC}}^{\mathrm{gen}}$ is the number of generated events in which the parton is emitted in this bin Monte Carlo studies show that the acceptance does not depend on quark flavour

The charge asymmetry is obtained by fitting the angular distribution to the function $f\left(\cos \theta_{\mathrm{h}}\right) \propto(1+$ $\cos ^{2} \theta_{\mathrm{h}}+\frac{8}{3} A_{\mathrm{h}}^{0} \cos \theta_{\mathrm{h}}$ ), where the asymmetry $A_{\mathrm{h}}^{0}$ is the only free parameter As mentioned earlier, the average weights are dependent on the asymmetry in the Monte Carlo, because of the different contributions of the various quark flavours The asymmetry within the Monte Carlo is adjusted such that the asymmetry measured from the Monte Carlo agrees with that observed in the data

As a cross-check, the asymmetry was calculated by simply counting the number of forward and backward events as

$A_{\mathrm{h}}=K \frac{N_{\mathrm{F}}-N_{\mathrm{B}}}{N_{\mathrm{F}}+N_{\mathrm{B}}}+\Delta A_{\mathrm{B}^{0} \overline{\mathrm{B}}^{0} \mathrm{mix}}$,

where the geometric correction factor $K$ appears because of the cut on $\left|\cos \theta_{\text {sph }}\right|$ and is calculated to be 1135

The relationships of the asymmetries $A_{\mathrm{h}}, A_{\mathrm{h}}^{0}$ and $A_{\mathrm{MC}}$ are indicated in fig $5 \mathrm{a}$ and the final fitted distribution is shown in fig 5 b Only statistical errors (eq (4)) for each $\cos \theta_{\mathrm{h}}$ bin are indicated in the figure The result using the weight function with $\kappa=1$ for all centre of mass energies is

$$
\begin{aligned}
& A_{\mathrm{h}}=0040 \pm 0004 \\
& \left(\chi^{2} / \mathrm{d} \text { of }=75 / 7\right)(\langle\sqrt{\mathrm{s}}\rangle=913 \mathrm{GeV}),
\end{aligned}
$$


Table 2

Resulting charge asymmetry from the fit for each energy region in the case of $\kappa=1$ and 0

\begin{tabular}{llllll}
\hline$\sqrt{s}[\mathrm{GeV}]$ & $\kappa=1$ & & $\kappa=0$ & \\
\cline { 2 - 3 } \cline { 5 - 6 } & $A_{\mathrm{h}} \pm \sigma_{\mathrm{A}}$ & $\chi^{2} / \mathrm{d}$ of & & $A_{\mathrm{h}} \pm \sigma_{\mathrm{A}}$ & $\chi^{2} / \mathrm{d}$ of \\
\hline $882-902(\langle\sqrt{s}\rangle=897)$ & $0034 \pm 0012$ & $176 / 7$ & & $0033 \pm 0013$ & $206 / 7$ \\
$9120-942(\langle\sqrt{s}\rangle=927)$ & $0045 \pm 0004$ & $43 / 7$ & & $044 \pm 0005$ & $51 / 7$ \\
all data $(\langle\sqrt{s}\rangle=913)$ & $0015 \pm 0009$ & $95 / 7$ & & $015 \pm 0010$ & $151 / 7$ \\
\hline
\end{tabular}
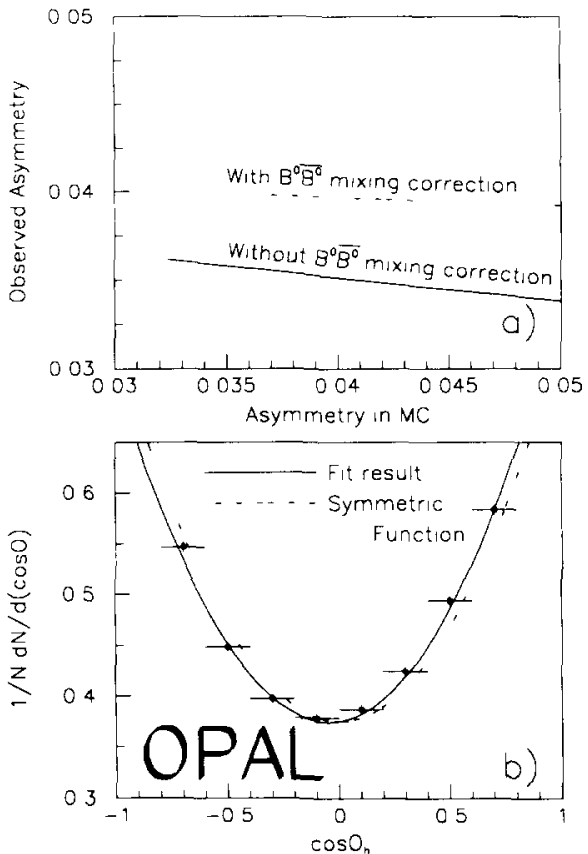

Fig 5 (a) The dependence of the observed charge asymmetry as a function of the charge asymmetry in the Monte Carlo events and (b) angular distribution of negatively charged jets for the whole data sample In the top figure (a), the dependence of the measured asymmetry on the asymmetry which is assumed in the Monte Carlo is shown with and without the correction for $\mathrm{B}^{0} \overline{\mathrm{B}^{0}}$ mixing by the dashed and solid lines, respectively In the bottom figure (b), the solid lines are the fit results without correction for the effect of $\mathbf{B}^{0} \overline{\mathrm{B}^{0}}$ mixing The dotted lines show the symmetric function, $\propto\left(1+\cos ^{2} \theta_{\mathrm{h}}\right)$ Only statistical errors for each $\cos \theta_{\mathrm{h}}$ bin are indicated

where this value includes the correction for $\mathrm{B}^{0} \overline{\mathrm{B}^{0}} \mathrm{mix}$ ing, as discussed in the previous section, and the errors are statistical only The results for the various energy ranges are listed in table 2 The poor $\chi^{2}$ of the fit
Table 3

Resultıng charge asymmetry by the simple countıng method for each energy region in the case of $\kappa=1$ and 0

\begin{tabular}{lll}
\hline$\sqrt{s}[\mathrm{GeV}]$ & \multicolumn{1}{l}{$A_{\mathrm{h}} \pm \sigma_{\mathrm{A}}$} & \\
\cline { 2 - 3 }$\kappa=1$ & $\kappa=0$ \\
\hline $882-902(\langle\sqrt{s}\rangle=897)$ & $0059 \pm 0016$ & $0059 \pm 0016$ \\
912 & $0043 \pm 0005$ & $0042 \pm 0005$ \\
$920-942(\langle\sqrt{s}\rangle=927)$ & $0019 \pm 0013$ & $0019 \pm 0013$ \\
all data $(\langle\sqrt{s}\rangle=913)$ & $0042 \pm 0005$ & $0042 \pm 0005$ \\
\hline
\end{tabular}

to the $\cos \theta_{\mathrm{h}}$ distribution of the lowest energy point is ascribed to a statistical fluctuation near $\cos \theta_{\mathrm{h}}=0$ It has little effect on the result of the fit, but does result in a discrepancy between the result obtained from fitting and that from simple countıng

When the data at different energy points were combined, the contributions were weighted by the number of selected events This is justified as $A_{\mathrm{h}}$ varies almost linearly with energy in the energy region measured within the sensitivity of this analysis

The counting method yields for all centre of mass energies

$A_{\mathrm{h}}=0042 \pm 0005 \quad(\langle\sqrt{s}\rangle=913 \mathrm{GeV})$

For the various energy ranges, we have also applied the simple counting method and the resulted asymmetries are listed in table 3 They are all in reasonable agreement with the above values for the fits Once again, a poor $\chi^{2}$ is obtained for the lowest energy point, due to the fluctuation mentioned above

As a check of the weight function method, event samples with different charge asymmetries at the parton level were created from JETSET Monte Carlo events with full detector simulation and were processed in the same way as the data A sample (430 127 
events) with a parton asymmetry of 004 resulted in a measured asymmetry of $0040 \pm 0004$ with $\chi^{2} / \mathrm{d}$ of $=23 / 7$, a sample with 008 asymmetry gave a measured asymmetry $0079 \pm 0004$ with $\chi^{2} / \mathrm{d}$ of $=21 / 7$ By counting forward and backward events, asymmetry values for these two samples were $0037 \pm 0005$ and $0077 \pm 0006$, respectively, both in good agreement with the values from the fits

As a second check of the weight function method, we have applied the same procedure to the data using only the charge information of the selected tracks $(\kappa=0)$ instead of weighting by the longitudinal momentum In this case, the fit result for all centre of mass energies is

$$
\begin{aligned}
& A_{\mathrm{h}}=0040 \pm 0004 \\
& \quad\left(\chi^{2} / \mathrm{d} \text { of }=90 / 7\right)((\sqrt{s}\rangle=913 \mathrm{GeV})
\end{aligned}
$$

The simple counting method yields in this case

$$
A_{\mathrm{h}}=0042 \pm 0005 \quad(\langle\sqrt{s}\rangle=913 \mathrm{GeV})
$$

The results of the fit and simple counting method for the various energy ranges are listed in table 2 and table 3 These results are almost identical to those obtained using $\kappa=1$ but with a slight increase in the statistical errors

\section{Systematic errors}

The main systematic error on the determination of the forward-backward charge asymmetry arises from the estımate of the averages of the weights, $\bar{W}_{\mathrm{F}}$ and $\bar{W}_{\mathrm{B}}$, because this relies on the validity of the Monte Carlo simulation Systematic errors due to inadequacies of the event generator were investigated by repeating the analysis for different values of the fragmentation parameters In the JETSET Monte Carlo, the following parameters were varied \pm 1 standard deviation from the OPAL optimised values [11]

$\Lambda_{\mathrm{QCD}} \Lambda$ value used in running $\alpha_{\mathrm{s}}$ for parton showers,

$\sigma_{\mathrm{q}}$ corresponding to the width in the gaussian transverse momentum distributions for primary hadrons,

$Q_{0}$ invariant mass cutoff parameter of parton showers, $a$ parameter of the Lund symmetric fragmentation function

The simple simulation program discussed in section 3 was used for this study The systematic errors obtained are listed in table 4 in the case of $\kappa=1$ and $\kappa=0$ Other fragmentation parameter in the JETSET Monte Carlo, $\gamma_{\mathrm{s}}$ and $V /(V+S)$, were also examined The parameter $\gamma_{s}$, the relative probability of picking up $s$ quarks from the vacuum in the fragmentation process, has been known to give a large effect on the jet charge determination The optımised value and allowed range at LEP energies [12] were used to estımate the systematic error The resulting uncertainty in the asymmetry was found to be \pm 00029 The parameter $V /(V+S)$ determining the vector over pseudoscalar production rate in the fragmentatıon, has been varied for $u$ and $d, s$, and $c$ and $b$ quarks independently around the JETSET defaults, taking into account theoretical limits The resultıng changes in the asymmetry are listed in table 4

We also checked the dependence on the fragmentation function for heavy quarks by comparing the Lund symmetric function [7] and the Peterson function [13] The Peterson fragmentation function characterises the fragmentation for heavy quarks, $c$ and $b$, with $\varepsilon_{\mathrm{c}}$ and $\varepsilon_{\mathrm{b}}=\left(m_{\mathrm{c}}^{2} / m_{\mathrm{b}}^{2}\right) \varepsilon_{\mathrm{c}}$ For the systematic error estımation, $\varepsilon_{c}$ was varied from 004 to 010 [14] We obtain an error of 00009

In order to further investigate the effects of the modeling of the fragmentation process, we compared the results obtained using a sample of 96721 events generated by the HERWIG Monte Carlo package [15] to calculate the average weights In this case, the difference in the measured asymmetry was found to be negligible

All the errors related to the event generator are summarised in table 4 and the total systematic error arısing from the event generator was estimated to be $\sigma_{\mathrm{A}}=00036$

As can be seen in fig 1, there are discrepancies between the $z_{\text {l }}$ distributions for data and Monte Carlo The simplest way to adjust the JETSET fragmentation parameters to take into account these distributions, in addition to the previous constraints, is a change in the parameter $\sigma_{\mathrm{q}}$ by approximately $2 \sigma$ Such a change can also account for the difference between data and Monte Carlo observed in fig $4 \mathrm{~b}$ The shift in the measured asymmetry resulting from this change of 
Table 4

Systematic errors on the measured asymmetry from fragmentation modelling

\begin{tabular}{|c|c|c|c|c|c|}
\hline \multirow[t]{2}{*}{ Parameter } & \multirow[t]{2}{*}{ Nominal value } & \multirow{2}{*}{\multicolumn{2}{|c|}{ Range }} & \multicolumn{2}{|l|}{$\sigma_{\mathbf{A}}$} \\
\hline & & & & $\kappa=1$ & $\kappa=0$ \\
\hline$\Lambda_{\mathrm{QCD}}$ & 029 & 028 & -031 & 00005 & 00004 \\
\hline$Q_{0}$ & 10 & 07 & -18 & 00011 & 00006 \\
\hline$\sigma_{\mathrm{q}}$ & 037 & 032 & -040 & 00009 & 00006 \\
\hline$a$ & 018 & 013 & -030 & 00010 & 00007 \\
\hline$\gamma_{\mathrm{s}}$ & 0285 & 0220 & -0320 & 00029 & 00030 \\
\hline$[V /(V+S)]_{\mathbf{u}, \mathrm{d}}$ & 050 & 030 & -075 & 00007 & 00007 \\
\hline$[V /(V+S)]_{\mathrm{s}}$ & 060 & 050 & -075 & 00003 & 00003 \\
\hline$[V /(V+S)]_{\mathrm{c}, \mathrm{b}}$ & 075 & 065 & -080 & 00004 & 00004 \\
\hline Peterson & 006 & 004 & -010 & 00009 & 00012 \\
\hline total & & & & 00036 & 00035 \\
\hline
\end{tabular}

$\sigma_{\mathrm{q}}$ is 0002 We add this in quadrature to the list of uncertainties discussed above

To study effects due to imperfections in the detector simulation, the momentum resolution of the simulated tracks has been changed up to two times the difference between the resolution for $\mu$-pair events in the data and the simulation In addition the track finding efficiency in the simulation has been lowered by $5 \%$ The maximal shift in the observed asymmetry is 0001 None of these changes can account for the discrepancies in the $z_{l}$ distributions displayed in fig 1

The effects of the sphericity cut were also investigated by changing the cut values Since the sphericity cut affects the rate of events with hard photon/gluon emission, this checks whether the Monte Carlo events correctly describe these radiative effects The observed asymmetry changed by less than 0002 when the sphericity cut was varied in the range of 010 014 The sphericity cut also changes the fraction of each quark flavour in the sample and therefore modifies the asymmetry With our cut of $S<012$, the $\mathrm{b} \overline{\mathrm{b}}$ fraction changes by only 0002 , and the effect on $A_{\mathrm{h}}$ was about 00002 We assign a systematic error of 0002 due to the sphericity cut

The sphericity axis cut, $\left|\cos \theta_{\mathrm{sph}}\right|<08$, can introduce a bias in the asymmetry measurement, because the sphericity axis is not exactly in the original parton direction, although the acceptance correction partıally compensates for this To check this effect, using the Monte Carlo sample, the sphericity axis distribution after the event selection and the acceptance cor- rection was compared with the original parton distribution With the $\left|\cos \theta_{\text {sph }}\right|<08$ cut, the sphericity axis distribution was in good agreement with that of partons This cut may also introduce an energy dependence of the photonic corrections, since the inltial state radiation modifies the angular distribution It was estımated by the ZFITTER program [2], and, in the energy range measured, the effect was less than 0001 Thus, the effect of the sphericity axis cut was estimated to be 0001

The cross-talk between the neighbouring bins in $\left|\cos \theta_{\text {sph }}\right|$ was found to be small because the bin size of at least $15^{\circ}$ was large compared to the mismatch in $\cos \theta_{\text {sph }}$ between the parton direction and the sphericity axis However, the cross-talk between the two bins at $\cos \theta_{\mathrm{sph}}=0$ makes the determination of forward/backward jets slightly ambiguous It is reflected in the errors of the average weights in those $\cos \theta_{\text {sph }}$ bins

The error due to the lack of higher order QED corrections ( $1 \mathrm{e}$, multiple photon emissions in the initial state) in the Monte Carlo generator was estımated by the ZFITTER program [2] This should be small because the effect of the initial state radiation is small on the $Z^{0}$ peak, and also because the energy dependence of the asymmetry is rather weak The contribution to the asymmetry was found to be much less than 0001

The weight function method is more sensitive to semi-leptonic decays of heavy quarks than to their hadronic decay modes Therefore, the semi-leptonic branching ratios for $\mathrm{b}$ and $\mathrm{c}$ quarks have been varied in opposite directions by $10 \%$, resultıng in a maximum 
Table 5

Systematic errors

\begin{tabular}{lrr}
\hline & \multicolumn{2}{c}{$\sigma_{\mathrm{A}}$} \\
\cline { 2 - 3 } & $\kappa=1$ & $\kappa=0$ \\
\hline fragmentation & 0004 & 0004 \\
shift in $\sigma_{\mathrm{q}}$ to adjust & & \\
$z_{l}$ distributions & 0002 & $<0002$ \\
detector effects & 0001 & 0001 \\
sphericity cut & 0002 & 0002 \\
$\left|\cos \theta_{\text {sph }}\right|<0$ 8 cut & 0001 & 0001 \\
initial state radiation & $<0001$ & $<0001$ \\
b, c semi-leptonic decay & & \\
branching ratio & $<0001$ & $<0001$ \\
Monte Carlo statistics & 0003 & 0004 \\
total & 0006 & 0007 \\
\hline
\end{tabular}

shift in the asymmetry of 00005

Due to the limited number of avallable Monte Carlo events with full detector simulation, the average weights fluctuate according to eq (5) The resulting error on the asymmetry was estımated to be 0003 from 430127 Monte Carlo events For $\kappa=0$, the error arising from the statistics of the number of Monte Carlo events was estımated to be 0004 This error increases because of the lower sensitivity of the weight function to the parton charge for $\kappa=0$

All the systematic errors were added in quadrature, and the total systematic error of the asymmetry was estımated to be 0006 (table 5)

\section{Charge asymmetry of the parton level and determination of the effective weak mixing angle}

Our final measured value for the charge asymmetry in hadronic $Z^{0}$ decays for the enture 1990 and 1991 OPAL data sample $(\langle\sqrt{s}\rangle=913 \mathrm{GeV})$ is

$$
\begin{aligned}
& A_{\mathrm{h}}=0040 \pm 0004 \text { (stat) } \pm 0006 \text { (syst) } \\
& \quad \pm 0002\left(\mathrm{~B}^{0} \overline{\mathrm{B}}^{0} \mathrm{mix}\right),
\end{aligned}
$$

including the effect of $\mathrm{B}^{0} \overline{\mathrm{B}^{0}}$ mixing If this asymmetry is compared with the theoretical prediction by the ZFITTER program [2], which includes photonic corrections of complete $O(\alpha)$, leading $O\left(\alpha^{2}\right)$ and soft photon exponentiation, it may be interpreted in terms of $\sin ^{2} \bar{\theta}_{\mathrm{w}}$
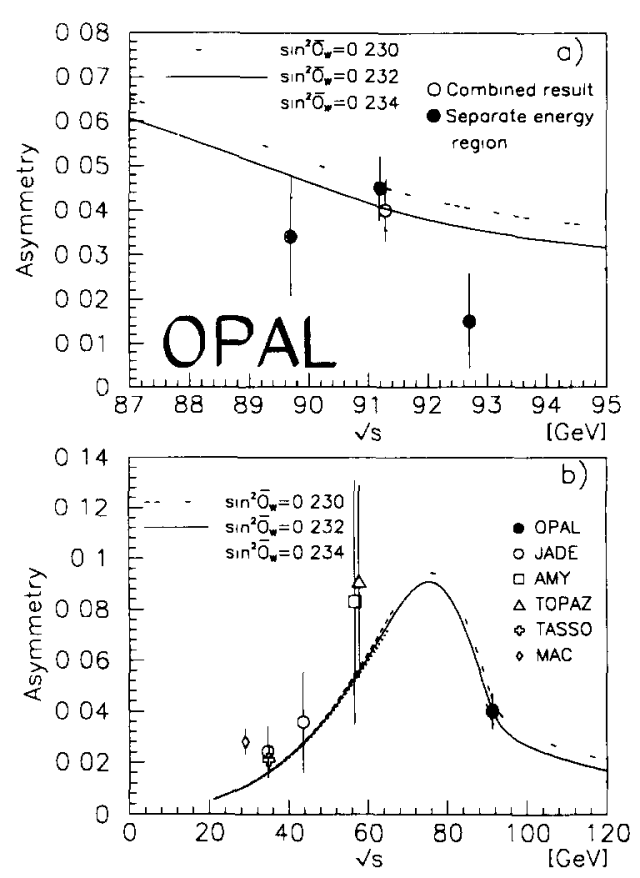

Fig 6 (a) The charge asymmetry corrected for the effect of $\mathrm{B}^{0} \overline{\mathrm{B}^{0}}$ mixing as a function of the centre of mass energy (closed circles) The open circle is the asymmetry for the whole data sample The lines are the standard model predictions with $\sin ^{2} \bar{\theta}_{\mathrm{W}}=0230,0232$, and 0234 (b) Comparison with lower energy results [3]

$$
\begin{aligned}
& \sin ^{2} \bar{\theta}_{\mathrm{W}}=02321 \pm 00017 \text { (stat) } \pm 00027 \text { (syst) } \\
& \pm 00009\left(\mathrm{~B}^{0} \overline{\mathrm{B}^{0}} \mathrm{mix}\right)
\end{aligned}
$$

As stated in the introduction, this is the average of the individual effective weak mixing angles for the five quark flavours, each weighted by the relative content of the appropriate quark, for a value of the top quark mass which reproduces the measured hadronic asymmetry Assuming a Higgs mass of $m_{\mathrm{H}}=100 \mathrm{GeV}$, the top quark mass $m_{\mathrm{t}}$ equivalent to the effective weak mixing angle result is

$m_{\mathrm{t}}=163_{-71}^{+50}(\text { stat })_{-127}^{+67}$ (syst $)_{-27}^{+21}\left(\mathrm{~B}^{0} \overline{\mathrm{B}^{0}} \mathrm{mIx}\right) \mathrm{GeV}$,

where the uncertainties correspond to those on $\sin ^{2} \bar{\theta}_{\mathrm{W}}$

The dependence on the mass of the Higgs was checked by varying the Higgs mass from $50 \mathrm{GeV}$ to $1000 \mathrm{GeV}$, and retuning the top mass to reproduce 
the measured asymmetry The effect on the average weak mixing angle was found to be negligibly small $(<00001)$ It should be noted that the effective weak mixing angles for the individual quark flavours are well within the quoted errors of the average effective weak mixing angle

In the context of the standard model, this result agrees well with the value of $m_{\mathrm{t}}=100_{-53}^{+78} \mathrm{GeV}$ [1], obtained from the OPAL measurement of the $Z^{0}$ line shape and lepton pair forward-backward asymmetry

A comparison of these results with the standard model prediction is shown in fig 6

\section{Conclusion}

We have measured the average quark forwardbackward charge asymmetry using 448942 hadronic $\mathrm{Z}^{0}$ decays collected with the OPAL detector at LEP We have used a weight function method and used the distributions of the longitudinal momenta for the leading charged particles to determine jet charge on a statistical basis

The observed charge asymmetry was obtained as $0040 \pm 0004$ (stat) \pm 0006 (syst) $\pm 0002\left(\mathbf{B}^{0} \overline{\mathbf{B}^{0}}\right.$ mix ), for the whole data sample where this result includes the correction for $\mathrm{B}^{0} \overline{\mathrm{B}^{0}}$ mixing In the framework of the standard model, the observed charge asymmetry corresponds to an effective weak mixing angle of $\sin ^{2} \bar{\theta}_{\mathrm{W}}=02321 \pm 00017$ (stat) \pm 00027 (syst ) $\pm 00009\left(\mathrm{~B}^{0} \overline{\mathrm{B}^{0}} \mathrm{mix}\right.$ ) Assuming a Higgs mass of $m_{\mathrm{H}}=100 \mathrm{GeV}$, the top mass corresponding to this effective weak mixing angle was determined to be $m_{\mathrm{t}}=163_{-71}^{+50}$ (stat $)_{-127}^{+67}$ (syst $)_{-27}^{+21}\left(\mathrm{~B}^{0} \overline{\mathrm{B}}^{0} \mathrm{mix}\right.$ ) $\mathrm{GeV}$ This agrees well with the value obtained from the $Z^{0}$ line shape and lepton parr forward-backward asymmetry

\section{Acknowledgement}

It is a pleasure to thank the SL Division for the efficient operation of the LEP accelerator and their continuing close cooperation with our experimental group In addition to the support staff at our own institutions we are pleased to acknowledge the Department of Energy, USA, National Science Foundation, USA,
Science and Engineering Research Council, UK, Natural Sciences and Engineerıng Research Council, Canada,

Israelı Ministry of Science, Minerva Gesellschaft, Japanese Minıstry of Education, Science and Culture (the Monbusho) and a grant under the Monbusho International Science Research Program, American Israelı B1-national Science Foundation, Direction des Sciences de la Matiere du Commissarıat à l'Energie Atomıque, France,

Bundesmınısterium fur Forschung und Technologie, FRG,

National Research Council of Canada, Canada,

A P Sloan Foundation and Junta Nacional de Investıgação Científica e Tecnológıca, Portugal

\section{References}

[1] OPAL Collab, MZ Akrawy et al, Z Phys C 52 (1991) 175

ALEPH Collab, D Decamp et al, Z Phys C 53 (1992) 1, DELPHI Collab, P Abrew et al, Nucl Phys B 367 (1991) 511 ,

L3 Collab, B Adeva et al, Z Phys C 51 (1991) 179, LEP Collab (ALEPH, DELPHI, L3 and OPAL), Phys Lett B 276 (1992) 243

[2] G Burgers and F Jegerlehner, in Z Physics at LEP1, CERN Yellow report 89-08, eds G Altarellı et al, Vol 1 (1989),

D Bardin et al, Comput Phys Commun 59 (1990) 303, Z Phys C 44 (1989) 493, Nucl Phys B 351 (1991) 1,

D Bardin et al, Phys Lett B 229 (1989) 405

[3] MAC Collab, W W Ash et al, Phys Rev Lett 58 (1987) 1080

JADE Collab, T Greenshaw et al, Z Phys C 42 (1989) 1,

TASSO Collab, W Braunschweıg et al, Z Phys C 41 (1988) 385,

AMY Collab, D Stuart et al, Phys Rev Lett 64 (1990) 983 ,

TOPAZ Collab, I Adachı et al, Phys Lett B 255 (1991) 613

[4] ALEPH Collab, D Decamp et al, Phys Lett B 259 (1991) 377,

DELPHI Collab P Abreu et al, CERN-PPE/91-211

[5] OPAL Collab, $\mathrm{K}$ Ahmet et al, Nucl Instrum Methods A305 (1991) 275

[6] J Allison et al, Comput Phys Commun 47 (1987) 55 , 
R Brun et al, GEANT 3, report DD/EE/84-1, CERN (1989)

[7] T Sjostrand, Comput Phys Commun 27 (1982) 243, 28 (1983) 229, 39 (1986) 347, 43 (1987) 367

[8] R Barlow, J Comp Phys 72 (1987) 202

[9] OPAL Collab, P D Acton et al, Phys Lett B 276 (1992) 379

[10] ARGUS Collab, H Albrecht et al, Phys Lett B 192 (1987) 245

CLEO Collab, M Artuso et al, Phys Rev Lett 62 (1989) 121
[11] OPAL Collab, M Z Akrawy et al, Z Phys C 47 (1990) 505

[12] OPAL Collab, G Alexander et al, Phys Lett B 264 (1991) 467,

OPAL Collab, P Acton et al , CERN-PPE/92-116

[13] C Peterson et al, Phys Rev D 27 (1983) 105

[14] J Chrin, Z Phys C 36 (1987) 163

[15] G Marchesını and B R Webber, Nucl Phys B 310 (1988) 461 